\title{
Coarse-Grid Selection for Parallel Algebraic Multigrid
}

\author{
A.J. Cleary \\ R. Falgout \\ V.E. Henson \\ J.E. Jones
}

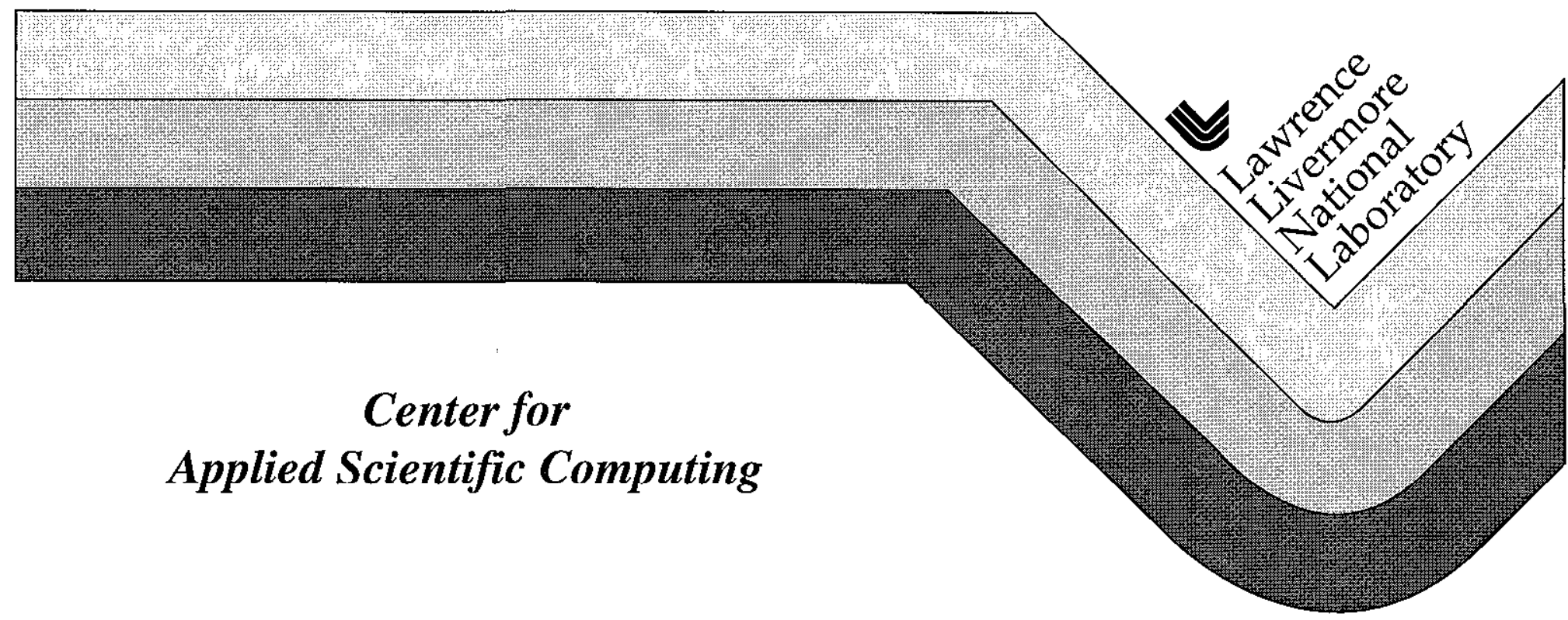

UCRL-JC-130893

June 1998 


\section{DISCLAIMER}

This document was prepared as an account of work sponsored by an agency of the United States Government Neither the United States Government nor the University of California nor any of their employees, makes any warranty, express or implied, or assumes any legal liability or responsibility for the accuracy, completeness, or usefulness of any information, apparatus, product, or process disclosed, or represents that its use would not infringe privately owned rights Reference herein to any specific commercial product, process, or service by trade name, trademark, manufacturer, or otherwise, does not necessarily constitute or imply its endorsement, recommendation, or favoring by the United States Government or the University of California The views and opinions of atthors expressed herein do not necessarily state or reflect those of the United States Government or the University of California, and shall not be used for advertising or product endorsement purposes

This is a preprint of a paper that was submitted to the Irregular 98 Conference, Berkeley, CA, August 9-11,1998 This preprint is made available with the understanding that it will not be cited or reproduced without the permission of the authors 


\title{
Coarse-grid Selection for Parallel Algebraic Multigrid
}

\author{
Andrew J Cleary, Robert D Falgout, Van Emden Henson, Jim E Jones \\ Center for Applied Scientific Computing, Law ence Livermore National Laboratory, \\ Liver more, CA
}

\begin{abstract}
Abstr act. The need to solve linear systems ar ising from problems posed on extr emely large, unstr uctured grids has sparked great inter est in parallelizing algebraic multign id (AMG) To date, however, no parallel AMG algorithms exist We introduce a parallel algorithm for the selection of coarse-grid points, a crucial component of AMG, based on modifications of certain parallel independent set algor ithms and the application of heur istics designed to insure the quality of the coarse grids A prototype serial version of the algorithm is implemented, and tests are conducted to deteımine its effect on multigrid convergence, and AMG complexity
\end{abstract}

\section{Introduction}

Since the introdurtion of algebraic multigi (AMG) in the 1980's $[4,2,3,5,19$, $16,18,17]$ the method has attıacted the attention of scientists needing to solve large problems posed on unstructured grids Recently, there has been a major surge of inter est in the field, due in large part to the need to solve increasingly larger systems, with hundreds of millions or billions of unknowns Most of the current research, however, focuses either on imploving the standard AMG algoithm $[9,7]$, or on dramatic new algebraic approaches [20,6] Little resear ch has been done on parallelizing AMG The sizes of the modern problems, however, dictate that large-scale parallel processing be employed

Methods for parallelizing geometric multignid methods have been known for some time [10], and most of the AMG algorithm can be parallelized using existing technology Indeed, much of the parallelization can be accomplished using tools readily available in packages such as PETSc or ISIS++ But, the heart of the AMG setup phase includes the coarse-grid selection process, which is inherently sequential in nature

In this note we introduce a parallel algorithm for selecting the coarse-grid points The algorithm is based on modifications of parallel independent set algolithms Also, we employ heuristics designed to insure the quality of the coarse grids A prototype serial code is implemented, and we examine the effect the algorithm has on the multigrid convergence properties

In Section 2 we outline the basic principles of AMG Section 3 describes our parallelization model and the underlying philosophy, while the details of the parallel algorithm are given in Section 4 Results of numerical experiments with the serial prototype are presented and analyzed in Section 5 In Section 6 we make concluding remarks and indicate directions for future research 


\section{Algebraic Multigrid}

We begin by outlining the basic principles of AMG Detailed explanations may be found in [17] Consider a problem of the form $A \mathbf{u}=\mathbf{f}$, where $A$ is an $n \times n$ matrix with entries $a_{i j}$ For AMG, a "grid" is simply a set of indices of the variables, so the original grid is denoted by $\Omega=\{1,2, \quad, n\}$ In any multigrid method, the central idea is that error e not eliminated by relaxation is eliminated by solving the residual equation $A \mathbf{e}=\mathbf{r}$ on a coarser guid, then interpolating e and using it to correct the fine-grid approximation The coarse-grid problem itself is solved by a recursive application of this method Proceeding through all levels, this is known as a multigrid cycle One purpose of AMG is to free the solver from dependence on geometry (which may not be easily accessible, if it is known at all) Hence, AMG fixes a relaxation method, and its main task is to determine a coarsening process that approximates error the relaxation cannot reduce

Using superscripts to indicate level number, where 1 denotes the finest level so that $A^{1}=A$ and $\Omega^{1}=\Omega$, the components that AMG needs are: "grids" $\Omega^{1} \supset \Omega^{2} \supset \supset \Omega^{M}$; grid operators $A^{1}, A^{2}, \quad, A^{M}$, inter polation operators $I_{k+1}^{k}, k=1,2, \quad M-1$, restriction operators $I_{k}^{k+1}, k=1,2, \quad M-1$, and a relaxation scheme for each level Once these components are defined, the recursively defined multigrid cycle is as follows:

Algorithm: $M V^{k}\left(\mathbf{u}^{k}, \mathbf{f}^{k}\right)$ The $\left(\mu_{1}, \mu_{2}\right)$ V-cycle

If $k=M$, set $\mathbf{u}^{M}=\left(A^{M}\right)^{-1} \mathbf{f}^{M}$

Other wise:

Relax $\mu_{1}$ times on $A^{k} \mathbf{u}^{k}=\mathrm{f}^{k}$

Pelform coarse grid consection

Set $\mathbf{u}^{k+1}=0, \mathbf{f}^{k+1}=I_{k}^{k+1}\left(\mathbf{f}^{k}-A^{k} \mathbf{u}^{k}\right)$

"Solve" on level $k+1$ with $M V^{k+1}\left(\mathbf{u}^{k+1}, \mathbf{f}^{k+1}\right)$

Correct the solution by $\mathbf{u}^{k} \leftarrow \mathbf{u}^{k}+I_{k+1}^{k} \mathbf{u}^{k+1}$

Relax $\nu_{2}$ times on $A^{k} \mathbf{u}^{k}=\mathbf{f}^{k}$

For this to work efficiently, two principles must be followed

P1: Enrors not efficiently reduced by relaxation must be well-approximated by the range of inter polation

P2: The coarse-grid problem must provide a good approximation to finegrid error in the range of inter polation

AMG satisfies $\mathbf{P} 1$ by automatically selecting the coarse grid and defining intexpolation, based solely on the algebraic equations of the system P2 is satisfied by defining restriction and the coarse-grid operator by the Galerkin for mulation [14]:

$$
I_{k}^{k+1}=\left(I_{k+1}^{k}\right)^{T} \quad \text { and } \quad A^{k+1}=I_{k}^{k+1} A^{k} I_{k+1}^{k}
$$

Selecting the AMG components is done in a separate preprocessing step 


\section{AMG Setup Phase:}

1 Set $k=1$

2 Partition $\Omega^{k}$ into disjoint sets $C^{k}$ and $F^{k}$

(a) Set $\Omega^{k+1}=C^{k}$

(b) Define intel polation $I_{k+1}^{k}$

3 Set $I_{k}^{k+1}=\left(I_{k+1}^{k}\right)^{T}$ and $A^{k+1}=I_{k}^{k+1} A^{k} I_{k+1}^{k}$

4 If $\Omega^{k+1}$ is small enough, set $M=k+1$ and stop Other wise, set $k=k+1$ and go to step 2

\subsection{Selecting Coarse Grids and Defining Interpolation}

Step 2 is the core of the AMG setup process The goal of the setup phase is to choose the set $C$ of coarse-grid points and, for each fine-grid point $i \in F \equiv \Omega-C$, a small set $C_{i} \subset C$ of inter polating points Interpolation is then of the form

$$
\left(I_{k+1}^{k} \mathbf{u}^{k+1}\right)_{i}=\left\{\begin{array}{cl}
\mathbf{u}_{i}^{k+1} & \text { if } i \in C, \\
\sum_{j \in C_{i}} \omega_{i j} \mathbf{u}_{j}^{k+1} & \text { if } i \in F
\end{array}\right.
$$

We do not detail the construction of the inter polation weights $\omega_{i j}$, instead referring the reader to [17] for details

An underlying assumption in AMG is that smooth en is is characterized by small residuals, that is, $A \mathbf{e} \approx 0$, which is the basis for choosing coarse grids and defining inter polation weights For simplicity of discussion here, assume that $A$ is a symmetric positive-definite $M$-matrix, with $a_{i i}>0, a_{i j} \leq 0$ for $j \neq i$, and $\sum a_{i j} \geq 0$

We say that point $i$ depends on point $j$ if $a_{i j}$ is "large" in some sense, and hence, to satisfy the $i$ th equation, the value of $u_{i}$ is affected more by the value of $u_{j}$ than by other variables Specifically, the set of dependencies of $i$ is defined by

$$
S_{i} \equiv\left\{j \neq i \quad-a_{i j} \geq \alpha \max _{k \neq i}\left(-a_{i k}\right)\right\}
$$

with $\alpha$ typically set to be 025 We also define the set $S_{i}^{T} \equiv\left\{j \quad i \in S_{j}\right\}$, that is, the set of points $j$ that depend on point $i$, and we say that $S_{i}^{T}$ is the set of influences of point $i$

A basic premise of AMG is that relaxation smoothes the enror in the direction of influence Hence, we may select $C_{i}=S_{i} \cap C$ as the set of interpolation points for $i$, and adhere to the following criterion while choosing $C$ and $F$ :

P3: For each $i \in F$, each $j \in S_{i}$ is either in $C$ or $S_{j} \cap C_{i} \neq \emptyset$

That is, if $i$ is a fine point, then the points influencing $i$ must either be coarse points or must themselves depend on the coarse points used to inter polate $u_{i}$

The coarse grid is chosen to satisfy two criteria We enforce P3 in order to insure good interpolation However, we wish to keep the size of the coarse-grid as small as possible, so we desire that 
P4: $C$ is a maximal set with the property that no $C$-point influences another $C$-point

It is not always possible to enforce both criteria Hence, we enforce P3 while using $\mathbf{P 4}$ as a guide in coar se-point selection

AMG employs a two-pass process, in which the grid is first "colored", providing a tentative $C / F$ choice Essentially, a point with the largest number of influences ("influence count") is colored as a $C$ point The points depending on this $C$ point are colored as $F$ points Other points influencing these $F$ points are more likely to be useful as $C$ points, so their influence count is increased The process is repeated until all points are either $C$ or $F$ points Next, a second pass is made, in which some $F$ points may be recolored as $C$ points to ensure that P3 is satisfied Details of the coarse-grid selection algorithm may be found in [17], while a recent study of the efficiency and robustness of the algotithm is detailed in [7]

Like many linear solvers, AMG is divided into two main phases, the setup phase and the solve phase Within each of these phases are certain tasks that must be parallelized to create a parallel AMG algorithm They are

- Setup phase:

- Selecting the coarse grid points, $\Omega^{k+1}$

- Construction of interpolation and uestriction operators, $I_{k+1}^{k}, I_{k}^{k+1}$

- Constructing the coarse-grid operator $A^{k+1}=I_{k}^{k+1} A^{k} I_{k}^{k+1}$

- Solve phase:

- Relaxation on $A^{k} \mathbf{u}^{k}=\mathbf{f}^{k}$

- Calculating the residual $\mathbf{r}^{k} \leftarrow \mathbf{f}^{k}-A^{k} \mathbf{u}^{k}$

- Computing the restriction $\mathbf{f}^{k+1}=I_{k}^{k+1} \mathrm{r}^{k}$

- Interpolating and correcting $u^{k} \leftarrow \mathbf{u}^{k}+I_{k+1}^{k} \mathbf{u}^{k+1}$

\section{Parallelization Model}

In this work we target massively parallel distributed memory architectures, though it is expected that the method will prove useful in other settings, as well Currently, most of the target platforms support shared memory within clusters of processors (typically of size 4 or 8 ), although for portability we do not utilize this feature We assume explicit message passing is used among the processors, and implement this with MPI [15] The equations and data are distributed to the processors using a domain-partitioning model This is natural for many problems of physics and engineering, where the physical domain is partitioned by subdomains The actual assignment to the processors may be done by the application code calling the solver, by the gridding program, or by a subsequent call to a graph partitioning package such as Metis [12] The domain-partitioning stıategy should not be confused with domain decomposition, which refers to a family of solution methods

We use object-oriented software design for parallel AMG One benefit of this design is that we can effectively employ kernels from other packages, such as 
PETSc [1] in several places throughout our code Internally, we focus on a matrix object that generalizes the features of "matrices" in widely-used packages We can write AMG-specific routines once, for a variety of matrix data structures, while avoiding the necessity of reinventing widely available routines, such as matrix-vec tor multiplication

Most of the required operations in the solve phase of AMG are standard, as are several of the core operations in the setup phase We list below the standard operations needed by AMG:

- Matrix-vector multiplication: used for residual calculation, for intex polation, and restriction (both use rectangular matrices, restriction multiplies by the transpose) Some packages provide all of the above, while others may have to be augmented, although the coding is straightfor ward in these cases

- Basic iterative methods used for the smoothing step Jacobi or scaled Jacobi are most common for parallel applications, but any iterative method provided in the parallel package could be applied

- Gathering/scattering processor boundary equations used in the constsuction of the inter polation operators and in the constr uction of coarse-grid oper ators via the Galerkin method Each processor must access "processor-boundary equations" stored on neighboring processors Because similar functionality is requir ed to implement additive Schwarz methods, parallel packages implementing such methods already provide tools that can be modified to fulfill this requirement

\section{Parallel Selection of Coarse Grids}

Designing a parallel algorithm for the selection of the coarse-grid points is the most difficult task in parallelizing AMG Classic al AMG uses a two-pass algorithm to implement the heuristics, P3 and P4, that assure gi id quality and control grid size In both passes, the algorithm is inherently sequential The first pass can be described as:

1) Find a point $j$ with maximal measure $w(j)$ Select $j$ as a $C$ point

2) Designate neighbors of $j$ as $F$ points, and update the measures of other near by points, using heuristics to insure grid quality Repeat steps 1) and 2) until all points are either $C$ or $F$ points

This algorithm is clearly unsuitable for parallelization, as updating of measures occurs after each $C$ point is selected The second pass of the classical AMG algorithm is designed to enforce $P 3$, although we omit the details and refer the reader to [17] We can satisfy $\mathbf{P 3}$ and eliminate the second pass through a simple modification of step 2)

Further, we may allow for parallelism by applying the following one-pass algorithm Begin by performing step 1) globally, selecting a set of $C$ points, $D$, and then perform step 2) locally, with each processor wor king on some por tion of the set $D$ With different criteria for selecting the set $D$, and armed with 
various heuristics for updating the neighbors in 2), a farnily of algorithms may be developed The overall framework is:

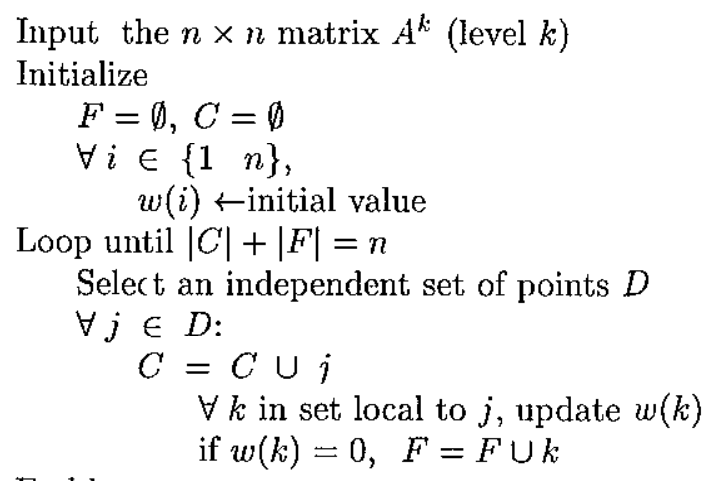

End loop

\subsection{Selection of the set $D$}

For the measure $w(i)$, we use $\left|S_{i}^{T}\right|+\sigma(i)$, the number of points influenced by the point $i$ plus a 1 andom number in $(0,1)$ The 1 andom number is used as a mechanism for breaking ties between points with the same number of influences The set $D$ is then selected using a modification of a parallel maximal independent set algorithm developed in $[13,11,8]$

A point $j$ will be placed in the set $D$ if $w(j)>w(k)$ for all $k$ that either influence or depend on $j$ By construction, this set will be independent While our implementation selects a maximal set of points possessing the requisite property, this is not necessary, and may not be optimal An important obser vation is that this step can be done entirely in parallel, provided each processor has access to the $w$ values for points with influences that cross its processor boundary

\section{Updating $w(k)$ of neighbors}

Describing the heuristics for updating $w(k)$ is best done in terms of graph theory We begin by defining $S$, the auxiliary influence matrix

$$
S_{i j}=\left\{\begin{array}{l}
1 \text { if } j \in S_{i}, \\
0 \text { other wise }
\end{array}\right.
$$

That is, $S_{i j}=1$ only if $i$ depends on $j$ The $i$ th row of $S$ gives the dependencies of $i$ while the $i$ th column of $S$ gives the influences of $i$ We can then form the directed graph of $S$, and observe that a directed edge from ver tex $i$ to vertex $j$ exists only if $S_{i j} \neq 0$ Notice that the directed edges point in the direction of dependence To update the $w(k)$ of neighbors, we apply the following pair of heur istics 
P5: Values at $C$ points are not interpolated, hence, neighbols that influence a $C$ point are less valuable as potential $C$ points themselves

P6: If $k$ and $j$ both depend on $c$, a given $C$ point, and $j$ influences $k$, then $j$ is less valuable as a potential $C$ point, since $k$ can be inter polated fiom $c$

The details of how these heuristics are implemented are:

$$
\begin{aligned}
\forall c \in D, & \\
\forall j \mid S_{c j} \neq 0, & \text { P5: } \\
w(j) \leftarrow w(j)-1 & \text { (each } j \text { that influences } c \text { ) } \\
S_{c j} \leftarrow 0 & \text { (decrement the measure) } \\
\forall j \mid S_{j c} \neq 0 & \text { (remove edge } c j \text { from the graph) } \\
S_{j c} \leftarrow 0 & \text { (each } j \text { that depends on } c \text { ) }, \\
\forall k \mid S_{k j} \neq 0, & \text { (remove edge } j c \text { fiom the graph) } \\
\text { if } S_{k c} \neq 0 & \text { (each } k \text { that } j \text { influences), } \\
w(j) \leftarrow w(j)-1 & \text { (if } k \text { depends on } c \text { ) }, \\
S_{k j} \leftarrow 0 & \text { (decrement the measure) }
\end{aligned}
$$

The heuristics have the effect of lowering the measure $w(k)$ for a set of neighbors of each point in $D$ As these measures are lowered, edges of the graph of $S$ are I emoved to indicate that cer tain influenc es have alr eady been taken into account Frequently the step $w(j) \rightarrow w(j)-1$ causes $\lfloor w(j)\rfloor=0$ When this occurs $j$ is flagged as an $F$ point

Once the heuristics have been applied for all the points in $D$, a global communication step is required, so that each processor has updated $w$ values for all neighbors of all their points The entire process is then repeated $C$ points are added by selecting a new set, $D$, from the ver tices that still have edges attached in the modified graph of $S$ This process continues until all $n$ points have either been selected as $C$ points or $F$ points

\section{$5 \quad$ Numerical Experiments}

To test its effect on convergence and algorithmic scalability, we include a sei ial implementation of the parallel coarsening algorithm in a standard sequential AMG solver Obviously, this does not test parallel efficiency, which must wait for a full parallel implementation of the entise AMG algorithm

Figure 1 shows the coarse grid selected by the parallel algorithm on a standard test problem, the 9-point Laplacian operator on a Iegular gi id This test is important because the grid selected by the standard sequential AMG algorithm 

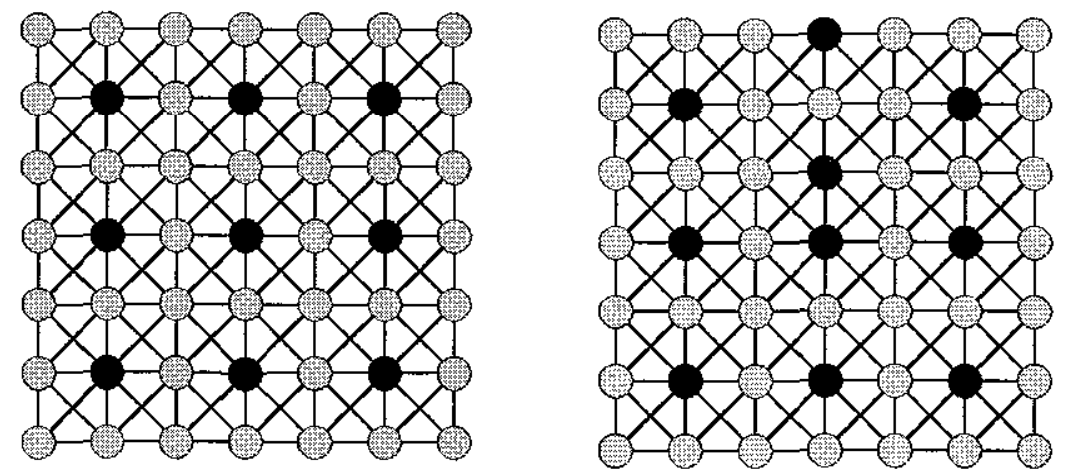

Fig 1 Coarse grids for the structured-grid 9-point Laplacian operator The dark circles are the $C$ points Left: Grid selected by the standard algorithm Right: Grid selected by the parallel algorithm
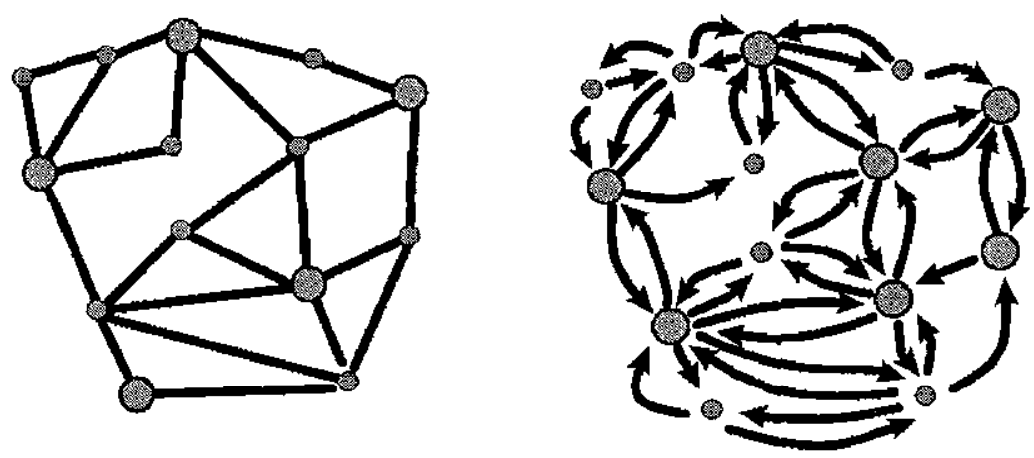

Fig. 2 Coarse grids for an unstructured grid The large circles are the $C$ points Left: Grid selected by the standard algorithm Right: Grid selected by the parallel algorithm Graph connectivity is shown on the left, while the full digraph is shown on the right

is also the optimal grid used in geometric multigrid for this problem Examining many such test problems on regular gxids, we find that the parallel coarsening algorithm typically produces coarse grids with $10-20 \%$ more $C$ points than the sequential algorithm On unstructured grids or complicated domains, this incr ease tends to be $40-50 \%$, as may be seen in the simple example displayed in Figure 2

The impact of the parallel coarsening algorithm on convergence and scalability is shown in two figures Figure 3 shows the convergence factor for the 9-point Laplacian oper ator on regular gi ids ranging in size from a few hundred to nearly a half million points Several different choices for the smoother and the parameter $\alpha$ are shown In Figure 4 the same tests are applied to the 9-point Laplacian operator for anisotropic grids, where the aspect ratios of the under- 


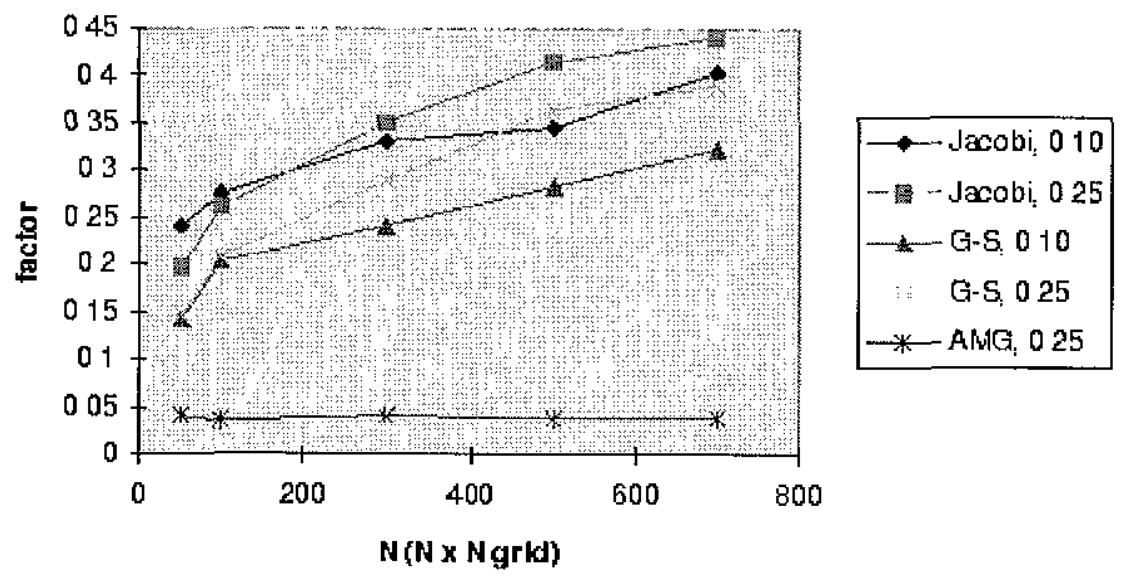

Fig 3 Convergence factors for parallel AMG for the 9-point Laplacian

lying quadilateral finite elements are extremely high In both figures, we see that convergence factors for the grids chosen by the parallel algotithm are significantly larger than standard AMG (shown as "AMG" in Figure 3, not shown in Figure 4), although the parallel algorithm still produce solutions in a reasonable number of iterations of more concern is that the convergence factors do not scale well with increasing problem size We believe that this may be caused by choosing too many coarse guid points at once, and that simple algorithmic modifications mentioned below may improve our results

Figure 5 shows the grid and operator complexities for the parallel algorithm applied to the 9-point Laplacian operator Grid complexity is the total number of grid points, on all grids, divided by the number of points on the original grid Operator complexity is the total number of non-zeros in all operators $A^{1}, A^{2}$, divided by the number of non-zeros in the original matrix Both the grid and operator complexities generated using by the parallel algorithm are essentially constant with increasing problem size While slightly larger than the complexities of the sequential giids, they never theless appear to be scalable

The fuamework described in Section 4 permits easy modification of the algorithm For example, one may alter the choice of the set $D$ of $C$ points We believe that the convergence factor degradation shown in our results may be due to selecting too many coarse grid points One possibility is to choose the minimal number of points in $D$, that is, one point per processor This amounts to running the sequential algorithm on each processor, and there a number of different ways to handle the interprocessor boundaries One possibility is to coarsen the processor boundary equations first, using a par allel MIS algorithm, and then treat each domain independently Another option is to I un the sequential algorithm on each processor ignoring the nodes on the boundary, and then patch up the 


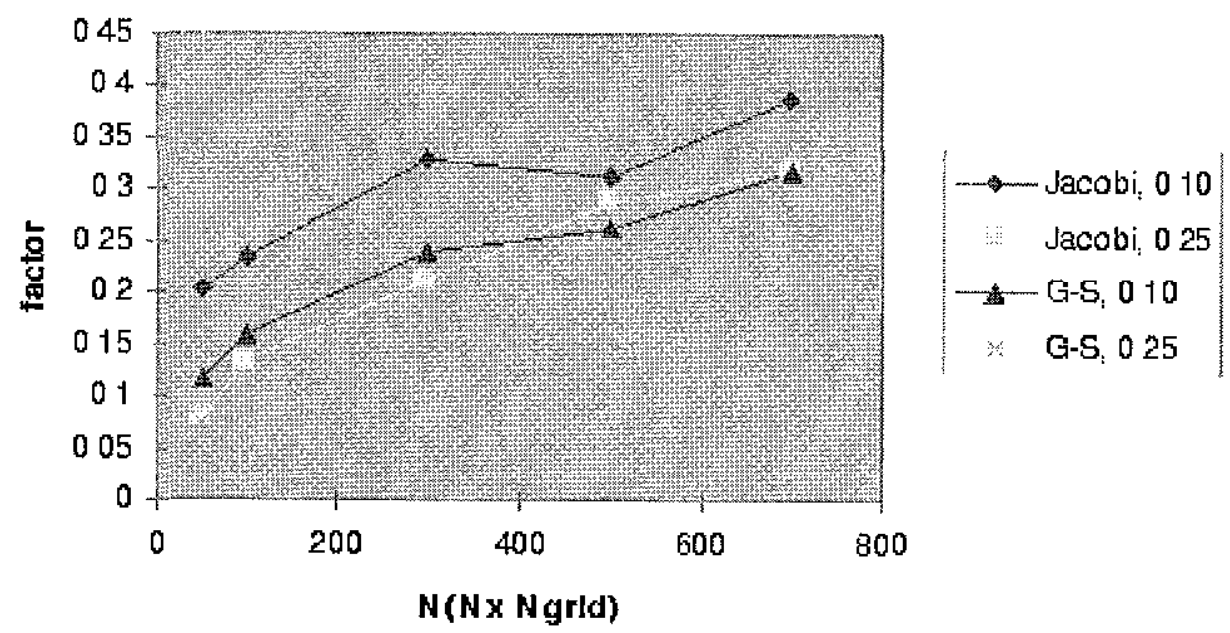

Fig. 4 Convergence rates for parallel $A M G$ for the anisotropic grid problem

grids on the processor boundaries

\section{Conclusions}

Modern massively parallel computing requires the use of scalable linear solvers such as multigrid For unstructured-giid problems, however, scalable solvers have not been developed Parallel AMG, when developed, promises to be such a solver AMG is divided into two main phases, the setup phase and the solve phase The solve phase can be parallelized using standard techniques common to most parallel multigrid codes However, the setup phase coarsening algorithm is inherently sequential in nature

We develop a family of algorithms for selecting coarse grids, and prototype one member of that family using a sequential code Tests with the prototype indicate that the quality of the selected coarse grids are sufficient to maintain constant complexity and to provide convergence even for diffic ult anisotropic problems However, convergence rates are higher than for standard AMG, and do not scale well with problem size We believe that this degradation may be caused by choosing too many coarse grid points at once, and that simple algorithmic modifications may improve our results Exploration of these algorithm variants is the subject of our cunrent research

\section{References}

1 S Balay, W Gropp, L C McInnes, and B Smith, Petsc 20 user's manual, Tech Rep ANL-95/11, Aıgonne National Laboratory, Nov 1995 


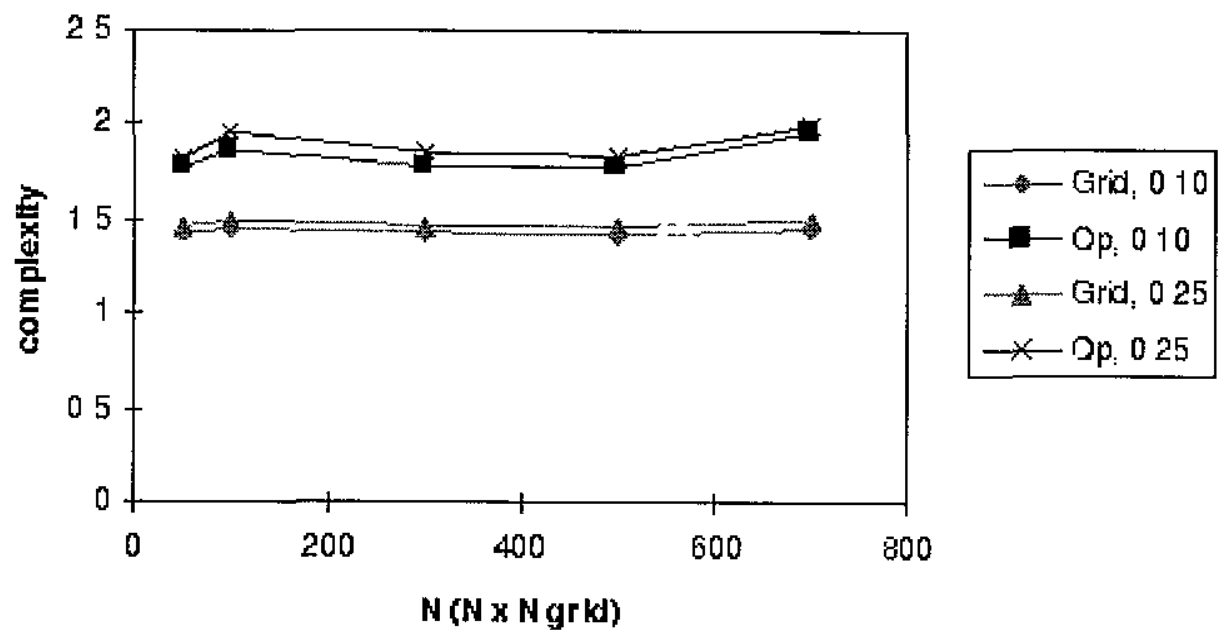

Fig. 5 Operator complexity for parallel $A M G$ on example problem

2 A BRandt, Algebraic multigrid theory The symmetric case, in Preliminary Proceedings for the Inter national Multigrid Conference, Copper Mountain, Color ado, April 1983

3 A BrandT, Algebraic multigrid theory: The symmetric case, Appl Math Comput , 19 (1986), pp 23-56

4 A BrandT, S F McCormick, And J W Ruge, Algebraic multigrid (AMG) for automatic multignid solutions with application to geodetic computations Report, Inst for Computational Studies, Fort Collins, Colo, October 1982

5 - Algebraic multigrid (AMG) for sparse matrix equations, in Sparsity and Its Applications, D J Evans, ed, Cambridge University Press, Cambridge, 1984

6 M Brezina, A J Cleary, R D Falgout, V E Henson, J E Jones, $T$ A ManteufFel, S F MCCormick, and J W Ruge, Algebraic multigrid based on element interpolation (AMGe) Submitted to the SIAM Jounal on Scientific Computing special issue on the Fifth Copper Mountain Conference on Iterative Methods, 1998

7 A J Cleary, $R$ D Falgout, V E Henson, J E Jones, $T$ a ManteufFEL, S F MCCORMICK, G N Miranda, AND J W Ruge, Robustness and scalability of algebraic multigrid Submitted to the SIAM Joun nal on Scientific Computing special issue on the Fifth Copper Mountain Conference on Iter ative Methods, 1998

8 R K Gjertsen, JR, M T Jones, and P E Plassman, Panallel heuristics for improved, balonced graph colorings, Joun nal of Parallel and Distributed Computing, 37 (1996), pp 171-186

9 G Golubovici AND C POPA, Interpolation and related coarsening techniques for the algebraic multigrid method, in Multignid Methods IV, Proceedings of the Four th European Multigi id Conference, Amsterdam, July 6-9, 1993, vol 116 of ISNM, Basel, 1994, Birkhäuser, pp 201-213 
$10 \mathrm{~J}$ E Jones And S F McCormick, Parallel multigrid methods, in Parallel Numerical Algorithms, D E Keys, A H Sameh, and V Venkatakrishnan, eds, Do1drecht, Netherlands, 1997, Kluwer Academic Publications

$11 \mathrm{M}$ T Jones AND P E PLASSMAN, A parallel graph coloring heuristic, SIAM Journal on Scientific Computing, 14 (1993), pp 654-669

12 G KARYPIS AND V KUMAR, A coarse-grain parallel multilevel $k$-way partitioning algorithm, in Proceedings of the 8th SIAM Conference on Parallel Processing for Scientific Computing, 1997

13 M LUBY, A simple parallel algorithm for the maximal independent set problem, SIAM Journal on Computing, 15 (1986), pp 1036-1053

$14 \mathrm{~S}$ F MCCORMICK, Multigritl methods for variational problems: general theory for the V-cycle, SIAM J Numer Anal, 22 (1985), pp 634-643

15 MPI FORUM, MPI: A message-passing interface standard, International J Supercomputing Applications, 8(3/4) (1994), pp 654-669

$16 \mathrm{~J}$ W RUGE AND K STÜBEN, Efficient solution of finite difference and finite element equations by algebraic multigrid $(A M G)$, in Multigxid Methods for Integral and Differential Equations, D J Paddon and H Holstein, eds, The Institute of Mathematics and its Applications Conference Series, Clarendon Press, Oxford, 1985, pp 169-212

17 - Algebraic multigrid (AMG), in Multignid Methods, S F McCormick, ed, vol 3 of Frontiers in Applied Mathematics, SIAM, Philadelphia, PA, 1987, pp 73130

$18 \mathrm{~K}$ STÜBEn, Algebraic multigrid (AMG) experiences and comparisons, Appl Math Comput, 13 (1983), pp 419-452

19 K Stüben, U Trottenberg, and K Witsch, Software development based on multigrid techniques, in Proc IFIP-Conference on PDE Software, Modules, Interfaces and Systems, B Enquist and T Smedsaas, eds, Sweden, 1983, Södel köping

20 P VANĚK, J MANDEL, AND M BREZINA, Algebraic multigrid based on smoothed aggregation for second and fourth order problems, Computing, 56 (1996), pp 179196

This article was processed using the $\mathrm{AT}_{\mathrm{E}} \mathrm{X}$ macro package with LLNCS style 


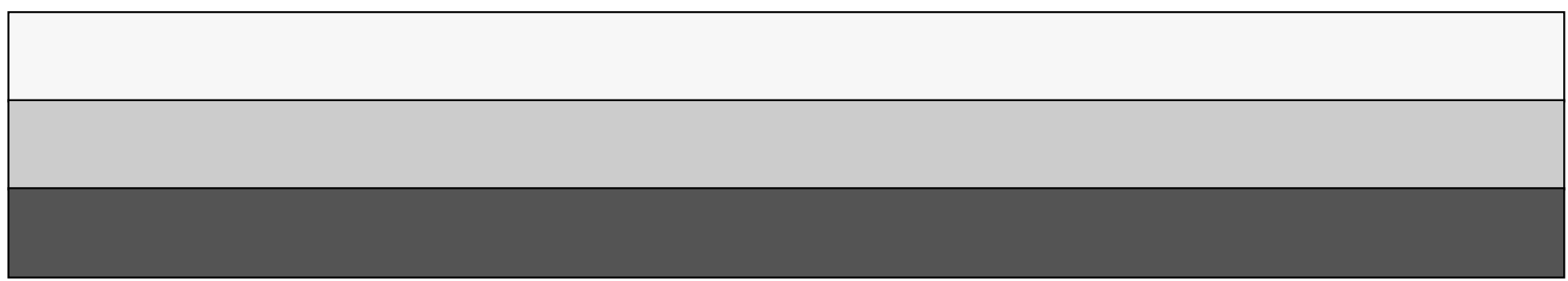

